\section{Australian Journal of \\ Crop Science}

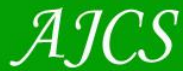

ISSN:1835-2707

AJCS 10(10):1474-1483 (2016)

DOI: 10.21475/ajcs.2016.10.10.p7786

\title{
Genetic variability among common black bean (Phaseolus vulgaris L.,) accessions in southern Brazil
}

Ivan Ricardo Carvalho*1, Maicon Nardino ${ }^{1}$, Mauricio Ferrari ${ }^{1}$, Alan Junior de Pelegrin ${ }^{1}$, Gustavo Henrique Demari ${ }^{2}$, Vinícius Jardel Szareski ${ }^{2}$, Diego Nicolau Follmann ${ }^{2}$, Carlos André Bahry ${ }^{3}$, Velci Queiróz de Souza ${ }^{2}$, Antonio Costa de Oliveira ${ }^{1}$, Luciano Carlos da Maia ${ }^{1}$

\author{
${ }^{1}$ Universidade Federal de Pelotas, CEP 96010-610, Capão do Leão, RS, Brasil \\ ${ }^{2}$ Universidade Federal de Santa Maria Campus de Frederico Westphalen, RS, Brasil \\ ${ }^{3}$ Universidade Tecnológica Federal do Paraná Campus Dois Vizinhos, PR, Brasil
}

\author{
*Corresponding authors:carvalho.irc@gmail.com
}

\begin{abstract}
To obtain superior genotypes, the use of natural genetic variability is essential, aiming to select parents that will make future crossings blocks. Therefore, the aim of this study was to determine the genetic variability and dissimilarity using multivariate analyzes in common black bean accessions native to southern Brazil. The experiment was conducted in the 2014/2015 in Frederico Westphalen - RS. The experimental design was augmented blocks (RCBD) with four repetitions (totally 149 treatments), in which 147 black common bean accessions were evaluated. Two commercial cultivars were used as witnesses: BRS Esplendor and BRS Supremo. The phenotypic traits such as days to flowering, plant height at flowering, days to maturity, plant height at maturity, insertion of the first pod, number of pods per plant, number of seeds per pod, mass of seeds per pod, number of branches, mass of seeds per plant, seed length, seed width, seed flattening, seed brightness, presence of halo, color of the halo were measured. The distributions of phenotypic frequencies indicate genetic variability among the 149 genotypes of common black bean. The Tocher optimization method presents the formation of eight groups of genotypes. The dendrogram formed by the standardized Euclidean distance was efficient in the stratification of the accessions for their genetic distance. The relative contribution evaluated by Singh method shows that the characters days to flowering and seed brightness are those that best discriminate the genotypes. The multivariate techniques of Tocher optimization and the standardized Euclidean distance show similar responses, proving to be viable tools for the choice of parents in a breeding program.
\end{abstract}

Keywords: genetic breeding; multivariate analyses; landraces; Phaseolus vulgaris $\mathrm{L}$.

Abbreviations: DTF_days to flowering; PHF_plant height at flowering; DTM_days to maturity; PHM_plant height at maturity; IFP_insertion of the first pod; NPP_number of pods per plant; NSP_number of seeds per pod; MSP_mass of seeds per pod; NB_number of branches; MSPP_mass of seeds per plant; SL_seed length; SW_seed width; SF_seed flattening; BRI_seed brightness; HAL_presence of halo; $\mathrm{CRH}_{-}$color of the halo; cm_centimeters; UPGMA_grouping of unweighed pairs basead on the arithmetric mean; MC_main components; CFA_subtropica; RCBD_ experimental design was of blocks augmented; m_meters; $\mathrm{m}^{2} \_s q u a r e$ meters; $\mathrm{kg} \mathrm{ha}^{-1}{ }_{\text {_ }}$ kilograms per hectare; V4_vegetative stage 4.

\section{Introduction}

The common black bean (Phaseolus vulgaris L.) is one of the major legumes intended for human consumption. It is grown in much of the Brazilian territory (Elias et al., 2007). The bean nutritional capacity is due to genotype, environment and genotype $\times$ environment interaction. The main components of nutrition are proteins, starch, sugar and vitamins (Flores et al., 2009). The genetic breeding of the species seeks genotypes that do not suffer from the environemental factors and interaction of genotype $\times$ environment. At the same time, they must have high production levels, cooking quality and high nutritional content (Carbonell et al., 2003).

The genetic breeding program of black bean has defined an ideotype with 35 days to start flowering (Silva et al., 2007), plants with stature higher than 50 centimeters (Horn et al., 2010), physiological maturity of up to 90 days (Elias et al., 2007), greater height of insertion of the first pod (Rocha et al., 2014), high number of pods per plant and seeds per pod (Kurek et al., 2001), reflecting high productivity (Ramos
Junior et al., 2005). Therefore, to obtain superior genotypes, it is essential to use the natural genetic variability to select efficient parents that will make future crossings programs. Hybridization between the genetically distant genotypes can enhance the genetic variability of the segregating populations and increase the chance of obtaining transgressive genotypes (Ramalho et al., 2012). Molecular techniques are used as effective tools to identify genes and alleles responsible for the traits of interest, selection of higher yielding genotypes, pyramiding gene, preparation of genetic maps and to identify the genetic variability of common black beans (Marin et al., 2005). Thus, understanding the genetic distance between the common black bean genotypes is crucial, where contrasting parents with additional and favorable traits are sought.

Among the multivariate analyses, the Tocher optimization method aims at subdividing the genotypes into groups, with the lowest genetic dissimilarity within and the highest dissimilarity among groups. The standardized Euclidean distance seeks through a set of observations to discriminate 
genotypes by forming groups with close phenotypes (Cruz et al., 2012). The methodology proposed by Singh (1981) can reveal the relative contribution of each character for discrimination of genotypes. The principal component analysis allows generating independent information and shows how many variables are needed to explain the genetic variation among genotypes (Cruz et al., 2012).

The aim of this study was to determine the genetic variability and dissimilarity by multivariate analyzes in common black bean accessions native to southern Brazil.

\section{Results and Discussion}

\section{Characterization of traits}

The descriptive analysis revealed the formation of different classes among the 149 genotypes for the traits days to flowering (DTF), plant height at flowering (PHF), days to maturity (DTM), plant height at maturity (PHM), insertion of the first pod (IFP), number of pods per plant (NPP), number of seeds per pod (NSP), mass of seeds per pod (MSP), number of branches (NB), mass of seeds per plant (MSPP), seed length (SL), seed width (SW), seed flattening (SF), seed brightness (BRI), presence of halo (HAL), and color of the halo $(\mathrm{CRH})$. The range of coefficients of variation of characters was $8.13 \%$ to $35.71 \%$, which shows adequate performance of the experiment.

\section{Performance of traits on the frequency of analysis}

The character days to flowering (DTF) revealed the formation of seven phenotypic classes with an amplitude of 25 to 55 days (Fig. 1). Classes of 40 and 45 DTF encompassed $74.5 \%$ of the genotypes. Genetic breeding programs aim to get early genotypes, with up to $35 \mathrm{DTF}$, requiring less water and nutrient resources. Given this, the selection of this character appears as the most viable way to define the common black bean cycle, controlled by a few genes with high heritability (Silva et al., 2007). Therefore, the selection for earliness can be proceded on the phenotypic classes of 25,30 and $35 \mathrm{DTF}$, consist of $23.75 \%$ of the studied genotypes.

The plant height at flowering (PHF) presented seven phenotypic classes, ranging from 15 to $85 \mathrm{~cm}$ (Fig. 1), wherein the classes of 15 and $25 \mathrm{~cm}$ consist of $70 \%$ of the genotypes. The agronomic ideotype favorable to the mechanical harvest must have plants with stature higher than $50.0 \mathrm{~cm}$ and erect (Horn et al., 2000). The character days to maturity (DTM) expressed eight phenotypic classes and amplitude from 72 to 114 DTM (Fig. 1). The class 102 DTM represents $48.3 \%$ of the genotypes. Research carried out with 45 common black bean genotypes determined the average length of 87.4 days (Elias et al., 2007). Therefore, the selection for earlier mature genotypes can occur by means of the phenotypic classes 72, 78 and 84 DTM.

Plant height at maturity (PHM) presented eight phenotypic classes ranging from 15 to $120 \mathrm{~cm}$ (Fig.1), wherein the class of $45 \mathrm{~cm}$ encompassed $36.9 \%$ of the genotypes. Studies to identify the best space arrangements for different bean genotypes showed final height of $54.1 \mathrm{~cm}$, concluding that the increase of this character have a negative impact on productivity (Morais et al., 2001). Thus, agronomically favorable genotypes can be obtained from the phenotypic classes of 45 to $60 \mathrm{~cm}$.

For the character insertion of the first pod (IFP), ten phenotypic classes with range of 3-30 cm were found (Fig.1).
The class of $15 \mathrm{~cm}$ stood out, with $22.8 \%$ of genotypes. Diallel analysis of bean genotypes advocate for genotypes with more IFP, and define that the genetic gain for this character was determined by the efficient choice of parents (Rocha et al., 2014). The selection to increment this character can occur in the classes 15, 18,21,24, 27 and $30 \mathrm{~cm}$.

The number of pods per plant (NPP) showed nine phenotypic classes, ranging 2 to 34 pods (Fig. 1). The class with six pods per plant accounted for $34.2 \%$ of genotypes. Multivariate analysis for 32 black bean genotypes indicated that the height and duration of the common black bean cycle are essential to increase the number of pods and the mass of seeds per plant (Coimbra et al., 2000). Increased productivity can be obtained by the phenotypic class with 34 pods per plant.

Regarding the number of seeds per pod (NSP), nine phenotypic classes were observed, with pods ranging from 1.2 to 6.0 seeds (Fig. 1). The class 4.2 seeds per pod included the largest fraction of the genotypes. The selection can be directed to the class with six seeds per pod. The mass of seeds per pod (MSP) revealed eight phenotypic classes ranging from 0.38 to 2.13 grams (Fig.1). However, 32.2\% of the genotypes were indexed in the class with 0.63 grams. A less contribution of NSP is attributed to the characteristics that contribute to more productive genotypes; however, more attention is given to the NPP (Kurek et al., 2001).

The number of branches (NB) presents seven phenotypic classes, with a range of 0.4 to 6.0 branches per plant (Fig. 2), and $32.2 \%$ of the genotypes reside in the lowest class. For the mass of seeds per plant (MSPP), nine phenotypic classes are formed, ranging from 3.0 to 27.0 grams of seed per plant (Fig. 2). The largest proportion of genotypes was located in the class with 6.0 grams, corresponding to $28.8 \%$ of genotypes. So, the selection of superior genotypes must be grounded in the characters mass of seeds per plant and number of seeds per pod. These are crucial to the productivity of the common black bean (Ramos Junior et al., 2005). Hence, obtaining more productive genotypes can be achieved in the phenotypic classes with greater magnitude of this character.

Seed length (SL) revealed eight phenotypic classes with a range of 6.0 to 13.0 millimeters. Of these, $32.2 \%$ of the genotypes were indexed in the class with $10.0 \mathrm{~mm}$ (Fig. 2). Seed width (SW) shows seven phenotypic classes, comprising from 3.9 to $8.1 \mathrm{~mm}$, in which $38.9 \%$ of the genotypes stood in the $6.3 \mathrm{~mm}$ class (Fig. 2). Silva (2005) classifies the shape of seeds by the ratio between length and width. The greater proportion of genotypes expressed ratio of 1.53 and the seed shape was assigned as elliptical.

As for the the visual characteristics determined by Silva (2005), we found that for the seed flattening (SF), $83.0 \%$ of the genotypes were classified as moderately filled seeds (class 2.0), 15\% as filled seeds (class 2.8) and $2.0 \%$ as flattened seeds (class 5.2) (Fig. 2). Regarding the brightness of the seed (BRI), $90 \%$ of the genotypes showed seeds with average brightness (class 2.03) and $10.0 \%$ had opaque seeds (class 0.83) (Fig. 2). The character presence of halo (HAL) shows that $93.0 \%$ of the genotypes do not have halo (class 1.02) (Fig. 2). For the color of halo (CRH), $99.0 \%$ of the genotypes express halo color similar to the rest of the seed (class 1.02).

\section{Performance of traits for grouping optimization Tocher}

The Tocher optimization method was performed for the 147 common black bean accessions and for two commercial 
Table 1. Additional information and origin of the common black bean access.

\begin{tabular}{|c|c|c|c|c|}
\hline $\begin{array}{l}\text { Access } \\
\text { identification }\end{array}$ & Genotypes & $\begin{array}{l}\begin{array}{l}\text { Population } \\
\text { source }\end{array} \\
\text { sol }\end{array}$ & City of origin & Geographic coordinates \\
\hline 1 & LMGPP1 & 1 & Campos Borges, RS, Brazil & \multirow{11}{*}{$28^{\circ} 52^{\prime} 31^{\prime \prime} \mathrm{S}$ and $53^{\circ} 00^{\prime} 55^{\prime \prime} \mathrm{W}$} \\
\hline 2 & LMGPP2 & 5 & Campos Borges, RS, Brazil & \\
\hline 3 & LMGPP3 & 6 & Campos Borges, RS, Brazil & \\
\hline 4 & LMGPP4 & 6 & Campos Borges, RS, Brazil & \\
\hline 5 & LMGPP5 & 8 & Campos Borges, RS, Brazil & \\
\hline 6 & LMGPP6 & 8 & Campos Borges, RS, Brazil & \\
\hline 7 & LMGPP7 & 8 & Campos Borges, RS, Brazil & \\
\hline 8 & LMGPP8 & 8 & Campos Borges, RS, Brazil & \\
\hline 9 & LMGPP9 & 9 & Campos Borges, RS, Brazil & \\
\hline 10 & LMGPP10 & 9 & Campos Borges, RS, Brazil & \\
\hline 11 & LMGPP11 & 10 & Palmeira das Missões, RS, Brazil & \\
\hline 12 & LMGPP12 & 12 & Palmeira das Missões, RS, Brazil & \multirow{19}{*}{$27^{\circ} 53^{\prime} 19^{\prime \prime S}$ and $53^{\circ} 18^{\prime} 19^{\prime \prime} \mathrm{W}$} \\
\hline 13 & LMGPP13 & 12 & Palmeira das Missões, RS, Brazil & \\
\hline 14 & LMGPP14 & 13 & Palmeira das Missões, RS, Brazil & \\
\hline 15 & LMGPP15 & 13 & Palmeira das Missões, RS, Brazil & \\
\hline 16 & LMGPP16 & 13 & Palmeira das Missões, RS, Brazil & \\
\hline 17 & LMGPP17 & 13 & Palmeira das Missões, RS, Brazil & \\
\hline 18 & LMGPP18 & 13 & Palmeira das Missões, RS, Brazil & \\
\hline 19 & LMGPP19 & 13 & Palmeira das Missões, RS, Brazil & \\
\hline 20 & LMGPP20 & 13 & Palmeira das Missões, RS, Brazil & \\
\hline 21 & LMGPP21 & 13 & Palmeira das Missões, RS, Brazil & \\
\hline 22 & LMGPP22 & 16 & Palmeira das Missões, RS, Brazil & \\
\hline 23 & LMGPP23 & 16 & Palmeira das Missões, RS, Brazil & \\
\hline 24 & LMGPP24 & 16 & Palmeira das Missões, RS, Brazil & \\
\hline 25 & LMGPP25 & 16 & Palmeira das Missões, RS, Brazil & \\
\hline 26 & LMGPP26 & 16 & Palmeira das Missões, RS, Brazil & \\
\hline 27 & LMGPP27 & 16 & Palmeira das Missões, RS, Brazil & \\
\hline 28 & LMGPP28 & 16 & Palmeira das Missões, RS, Brazil & \\
\hline 29 & LMGPP29 & 20 & Palmeira das Missões, RS, Brazil & \\
\hline 30 & LMGPP30 & 20 & Palmeira das Missões, RS, Brazil & \\
\hline
\end{tabular}

\begin{tabular}{|c|c|c|c|c|}
\hline $\begin{array}{l}\text { Access } \\
\text { identification }\end{array}$ & Genotypes & $\begin{array}{l}\text { Population } \\
\text { source }\end{array}$ & City of origin & Geographic coordinates \\
\hline 31 & LMGPP31 & 21 & Santa Rosa, RS, Brazil & \\
\hline 32 & LMGPP32 & 24 & Santa Rosa, RS, Brazil & \\
\hline 33 & LMGPP33 & 24 & Santa Rosa, RS, Brazil & \\
\hline 34 & LMGPP34 & 25 & Santa Rosa, RS, Brazil & \\
\hline 35 & LMGPP35 & 25 & Santa Rosa, RS, Brazil & \\
\hline 36 & LMGPP36 & 38 & Santa Rosa, RS, Brazil & \\
\hline 37 & LMGPP37 & 39 & Santa Rosa, RS, Brazil & \\
\hline 38 & LMGPP38 & 39 & Santa Rosa, RS, Brazil & \\
\hline 39 & LMGPP39 & 39 & Santa Rosa, RS, Brazil & \\
\hline 40 & LMGPP40 & 40 & Santa Rosa, RS, Brazil & \\
\hline 41 & LMGPP41 & 40 & Santa Rosa, RS, Brazil & \\
\hline 42 & LMGPP42 & 40 & Santa Rosa, RS, Brazil & \\
\hline 43 & LMGPP43 & 40 & Santa Rosa, RS, Brazil & \\
\hline 44 & LMGPP44 & 41 & Santa Rosa, RS, Brazil & $27^{\circ} 52^{\prime} 16^{\prime \prime} \mathrm{S}$ and $54^{\circ} 28^{\prime} 55^{\prime \prime} \mathrm{W}$ \\
\hline 45 & LMGPP45 & 41 & Santa Rosa, RS, Brazil & \\
\hline 46 & LMGPP46 & 41 & Santa Rosa, RS, Brazil & \\
\hline 47 & LMGPP47 & 41 & Santa Rosa, RS, Brazil & \\
\hline 48 & LMGPP48 & 41 & Santa Rosa, RS, Brazil & \\
\hline 49 & LMGPP49 & 41 & Santa Rosa, RS, Brazil & \\
\hline 50 & LMGPP50 & 41 & Santa Rosa, RS, Brazil & \\
\hline 51 & LMGPP51 & 41 & Santa Rosa, RS, Brazil & \\
\hline 52 & LMGPP52 & 47 & Santa Rosa, RS, Brazil & \\
\hline 53 & LMGPP53 & 49 & Santa Rosa, RS, Brazil & \\
\hline 54 & LMGPP54 & 49 & Santa Rosa, RS, Brazil & \\
\hline 55 & LMGPP55 & 49 & Santa Rosa, RS, Brazil & \\
\hline 56 & LMGPP56 & 49 & Santa Rosa, RS, Brazil & \\
\hline 57 & LMGPP57 & 49 & Santa Rosa, RS, Brazil & \\
\hline 58 & LMGPP58 & 50 & Pejuçara, RS, Brazil & \\
\hline 59 & LMGPP59 & 50 & Pejuçara, RS, Brazil & $28^{\circ} 25^{\prime} 24^{\prime \prime} \mathrm{S}$ and $53^{\circ} 39^{\prime} 21^{\prime \prime} \mathrm{W}$ \\
\hline 60 & LMGPP60 & 52 & Pejuçara, RS, Brazil & \\
\hline
\end{tabular}

Continue. 


\begin{tabular}{|c|c|c|c|c|}
\hline Access identification & Genotypes & $\begin{array}{l}\begin{array}{l}\text { Population } \\
\text { source }\end{array} \\
\end{array}$ & City of origin & Geographic coordinates \\
\hline 61 & LMGPP61 & 52 & Pejuçara, RS, Brazil & $28^{\circ} 25^{\prime} 24^{\prime \prime} \mathrm{S}$ and $53^{\circ} 39^{\prime} 21^{\prime \prime} \mathrm{W}$ \\
\hline 62 & LMGPP62 & 55 & Pejuçara, RS, Brazil & \\
\hline 63 & LMGPP63 & 55 & Pejuçara, RS, Brazil & \\
\hline 64 & LMGPP64 & 55 & Pejuçara, RS, Brazil & \\
\hline 65 & LMGPP65 & 58 & Pejuçara, RS, Brazil & \\
\hline 66 & LMGPP66 & 59 & Pejuçara, RS, Brazil & \\
\hline 67 & LMGPP67 & 64 & Pejuçara, RS, Brazil & \\
\hline 68 & LMGPP68 & 64 & Pejuçara, RS, Brazil & \\
\hline 69 & LMGPP69 & 64 & Pejuçara, RS, Brazil & \\
\hline 70 & LMGPP70 & 64 & Pejuçara, RS, Brazil & \\
\hline 71 & LMGPP71 & 64 & Pejuçara, RS, Brazil & \\
\hline 72 & LMGPP72 & 64 & Pejuçara, RS, Brazil & \\
\hline 73 & LMGPP73 & 64 & Pejuçara, RS, Brazil & \\
\hline 74 & LMGPP74 & 64 & Pejuçara, RS, Brazil & \\
\hline 75 & LMGPP75 & 64 & Pejuçara, RS, Brazil & \\
\hline 76 & LMGPP76 & 64 & Pejuçara, RS, Brazil & \\
\hline 77 & LMGPP77 & 64 & Pejuçara, RS, Brazil & \\
\hline 78 & LMGPP78 & 64 & Pejuçara, RS, Brazil & \\
\hline 79 & LMGPP79 & 64 & Pejuçara, RS, Brazil & \\
\hline 80 & LMGPP80 & 64 & Pejuçara, RS, Brazil & \\
\hline 81 & LMGPP81 & 64 & Pejuçara, RS, Brazil & \\
\hline 82 & LMGPP82 & 64 & Pejuçara, RS, Brazil & \\
\hline 83 & LMGPP83 & 64 & Pejuçara, RS, Brazil & \\
\hline 84 & LMGPP84 & 64 & Pejuçara, RS, Brazil & \\
\hline 85 & LMGPP85 & 64 & Pejuçara, RS, Brazil & \\
\hline 86 & LMGPP86 & 64 & Pejuçara, RS, Brazil & \\
\hline 87 & LMGPP87 & 64 & Pejuçara, RS, Brazil & \\
\hline 88 & LMGPP88 & 64 & Pejuçara, RS, Brazil & \\
\hline 89 & LMGPP89 & 64 & Pejuçara, RS, Brazil & \\
\hline 90 & LMGPP90 & 64 & Pejuçara, RS, Brazil & \\
\hline
\end{tabular}

\begin{tabular}{|c|c|c|c|c|}
\hline $\begin{array}{l}\text { Access } \\
\text { identification }\end{array}$ & Genotypes & $\begin{array}{l}\text { Population } \\
\text { source }\end{array}$ & City of origin & Geographic coordinates \\
\hline 61 & LMGPP61 & 52 & Pejuçara, RS, Brazil & \multirow{30}{*}{$28^{\circ} 25^{\prime} 24^{\prime \prime} \mathrm{S}$ and $53^{\circ} 39^{\prime} 21^{\prime \prime W}$} \\
\hline 62 & LMGPP62 & 55 & Pejuçara, RS, Brazil & \\
\hline 63 & LMGPP63 & 55 & Pejuçara, RS, Brazil & \\
\hline 64 & LMGPP64 & 55 & Pejuçara, RS, Brazil & \\
\hline 65 & LMGPP65 & 58 & Pejuçara, RS, Brazil & \\
\hline 66 & LMGPP66 & 59 & Pejuçara, RS, Brazil & \\
\hline 67 & LMGPP67 & 64 & Pejuçara, RS, Brazil & \\
\hline 68 & LMGPP68 & 64 & Pejuçara, RS, Brazil & \\
\hline 69 & LMGPP69 & 64 & Pejuçara, RS, Brazil & \\
\hline 70 & LMGPP70 & 64 & Pejuçara, RS, Brazil & \\
\hline 71 & LMGPP71 & 64 & Pejuçara, RS, Brazil & \\
\hline 72 & LMGPP72 & 64 & Pejuçara, RS, Brazil & \\
\hline 73 & LMGPP73 & 64 & Pejuçara, RS, Brazil & \\
\hline 74 & LMGPP74 & 64 & Pejuçara, RS, Brazil & \\
\hline 75 & LMGPP75 & 64 & Pejuçara, RS, Brazil & \\
\hline 76 & LMGPP76 & 64 & Pejuçara, RS, Brazil & \\
\hline 77 & LMGPP77 & 64 & Pejuçara, RS, Brazil & \\
\hline 78 & LMGPP78 & 64 & Pejuçara, RS, Brazil & \\
\hline 79 & LMGPP79 & 64 & Pejuçara, RS, Brazil & \\
\hline 80 & LMGPP80 & 64 & Pejuçara, RS, Brazil & \\
\hline 81 & LMGPP81 & 64 & Pejuçara, RS, Brazil & \\
\hline 82 & LMGPP82 & 64 & Pejuçara, RS, Brazil & \\
\hline 83 & LMGPP83 & 64 & Pejuçara, RS, Brazil & \\
\hline 84 & LMGPP84 & 64 & Pejuçara, RS, Brazil & \\
\hline 85 & LMGPP85 & 64 & Pejuçara, RS, Brazil & \\
\hline 86 & LMGPP86 & 64 & Pejuçara, RS, Brazil & \\
\hline 87 & LMGPP87 & 64 & Pejuçara, RS, Brazil & \\
\hline 88 & LMGPP88 & 64 & Pejuçara, RS, Brazil & \\
\hline 89 & LMGPP89 & 64 & Pejuçara, RS, Brazil & \\
\hline 90 & LMGPP90 & 64 & Pejuçara, RS, Brazil & \\
\hline
\end{tabular}




\begin{tabular}{|c|c|c|c|c|c|c|}
\hline \multicolumn{2}{|c|}{ Access identification } & \multicolumn{2}{|c|}{ Genotypes } & $\begin{array}{l}\text { Population } \\
\text { source }\end{array}$ & City of origin & \multirow[t]{2}{*}{ Geographic coordinates } \\
\hline 91 & & \multicolumn{2}{|c|}{ LMGPP91 } & 64 & Pejuçara, RS, Brazil & \\
\hline 92 & & \multicolumn{2}{|c|}{ LMGPP92 } & 67 & \multicolumn{2}{|l|}{ Braga, RS, Brazil } \\
\hline 93 & & \multicolumn{2}{|c|}{ LMGPP93 } & 68 & \multicolumn{2}{|l|}{ Braga, RS, Brazil } \\
\hline 94 & & \multicolumn{2}{|c|}{ LMGPP94 } & 68 & Braga, RS, Brazil & \\
\hline 95 & & LM & PP95 & 69 & Braga, RS, Brazil & \\
\hline 96 & & LM & PP96 & 69 & Braga, RS, Brazil & \\
\hline 97 & & LM & PP97 & 69 & Braga, RS, Brazil & \\
\hline 98 & & LM & PP98 & 69 & Braga, RS, Brazil & \\
\hline 99 & & LM & PP99 & 72 & Braga, RS, Brazil & $2 / 3 / 16 \mathrm{~s}$ and $53441 / \mathrm{w}$ \\
\hline 100 & & LM & PP100 & 72 & Braga, RS, Brazil & \\
\hline 101 & & LM & PP101 & 72 & Braga, RS, Brazil & \\
\hline 102 & & LM & PP102 & 72 & Braga, RS, Brazil & \\
\hline 103 & & $\mathrm{LM}$ & PP103 & 72 & Braga, RS, Brazil & \\
\hline 104 & & LM & PP104 & 72 & Braga, RS, Brazil & \\
\hline 105 & & $\mathrm{LM}$ & PP105 & 72 & Braga, RS, Brazil & \\
\hline 106 & & LM & PP106 & 73 & Cruz Alta, RS, Brazil & \\
\hline 107 & & LM & PP107 & 73 & Cruz Alta, RS, Brazil & \\
\hline 108 & & LM & PP108 & 73 & Cruz Alta, RS, Brazil & \\
\hline 109 & & LM & PP109 & 73 & Cruz Alta, RS, Brazil & \\
\hline 110 & & LM & PP110 & 73 & Cruz Alta, RS, Brazil & \\
\hline 111 & & LM & PP111 & 73 & Cruz Alta, RS, Brazil & \\
\hline 112 & & LM & PP112 & 73 & Cruz Alta, RS, Brazil & \\
\hline 113 & & LM & PP113 & 74 & Cruz Alta, RS, Brazil & $28^{\circ} 38^{\prime} 22^{\prime \prime} \mathrm{S}$ and $53^{\circ} 36^{\prime} 22^{\prime \prime} \mathrm{W}$ \\
\hline 114 & & $\mathrm{LM}$ & PP114 & 74 & Cruz Alta, RS, Brazil & \\
\hline 115 & & LM & PP115 & 74 & Cruz Alta, RS, Brazil & \\
\hline 116 & & LM & PP116 & 74 & Cruz Alta, RS, Brazil & \\
\hline 117 & & LM & PP117 & 74 & Cruz Alta, RS, Brazil & \\
\hline 118 & & LM & PP118 & 74 & Cruz Alta, RS, Brazil & \\
\hline 119 & & LM & PP119 & 74 & Cruz Alta, RS, Brazil & \\
\hline 120 & & LM & PP120 & 74 & Cruz Alta, RS, Brazil & \\
\hline $\begin{array}{l}\text { Access } \\
\text { Identification }\end{array}$ & Genoty & & $\begin{array}{l}\text { Population } \\
\text { source }\end{array}$ & City of ori & & Geographic coordinates \\
\hline 121 & LMGP & & 74 & Cruz Alta & S, Brazil & \\
\hline 122 & LMGP & & 74 & Cruz Alta & S, Brazil & \\
\hline 123 & LMGP & & 74 & Cruz Alta & S, Brazil & \\
\hline 124 & LMGP & & 75 & Cruz Alta, & S, Brazil & \\
\hline 125 & LMGP & & 75 & Cruz Alta & S, Brazil & \\
\hline 126 & LMGP & 26 & 75 & Cruz Alta & S, Brazil & $28^{\circ} 38^{\prime} 22^{\prime \prime} \mathrm{S}$ and $53^{\circ} 36^{\prime} 22^{\prime \prime} \mathrm{W}$ \\
\hline 127 & LMGP & & 75 & Cruz Alta & S, Brazil & \\
\hline 128 & LMGP & & 75 & Cruz Alta & S, Brazil & \\
\hline 129 & LMGP & & 75 & Cruz Alta & S, Brazil & \\
\hline 130 & LMGP & & 75 & Cruz Alta & S, Brazil & \\
\hline 131 & LMGP & & 75 & Cruz Alta & S, Brazil & \\
\hline 132 & LMGP & & 76 & Fortaleza & s Valos, RS, Brazil & \\
\hline 133 & LMGP & & 76 & Fortaleza & s Valos, RS, Brazil & \\
\hline 134 & LMGP & & 76 & Fortaleza & s Valos, RS, Brazil & \\
\hline 135 & LMGP & & 76 & Fortaleza & s Valos, RS, Brazil & \\
\hline 136 & LMGP & & 76 & Fortaleza & s Valos, RS, Brazil & \\
\hline 137 & LMGP & & 76 & Fortaleza & s Valos, RS, Brazil & $28 \quad 46$ 60 $\mathrm{s}$ and 53 13 $24 \mathrm{~W}$ \\
\hline 138 & LMGP & & 76 & Fortaleza & s Valos, RS, Brazil & \\
\hline 139 & LMGP & & 76 & Fortaleza & s Valos, RS, Brazil & \\
\hline 140 & LMGP & 40 & 76 & Fortaleza & s Valos, RS, Brazil & \\
\hline 141 & LMGP & & 76 & Fortaleza & s Valos, RS, Brazil & \\
\hline 142 & LMGP & 42 & 8 & Campos B & ges, RS, Brazil & \\
\hline 143 & LMGP & & 8 & Campos B & ges, RS, Brazil & \\
\hline 144 & LMGP & & 8 & Campos B & ges, RS, Brazil & \\
\hline 145 & LMGP & & 8 & Campos B & ges, RS, Brazil & $28^{\circ} 52^{\prime} 31^{\prime \prime} \mathrm{S}$ and $53^{\circ} 00^{\prime} 55^{\prime \prime} \mathrm{W}$ \\
\hline 146 & LMGP & 46 & 8 & Campos B & ges, RS, Brazil & \\
\hline 147 & LMGP & & 8 & Campos B & ges, RS, Brazil & \\
\hline 148 & $\begin{array}{l}\text { BRS } \\
\text { Esplen }\end{array}$ & & $\begin{array}{l}\text { Commercial } \\
\text { cultivars } \\
\text { witnesses }\end{array}$ & $\cdot$ & & $\cdot$ \\
\hline 149 & BRS S & remo & $\begin{array}{l}\text { Commercial } \\
\text { cultivars } \\
\text { witnesses }\end{array}$ & . & & . \\
\hline
\end{tabular}



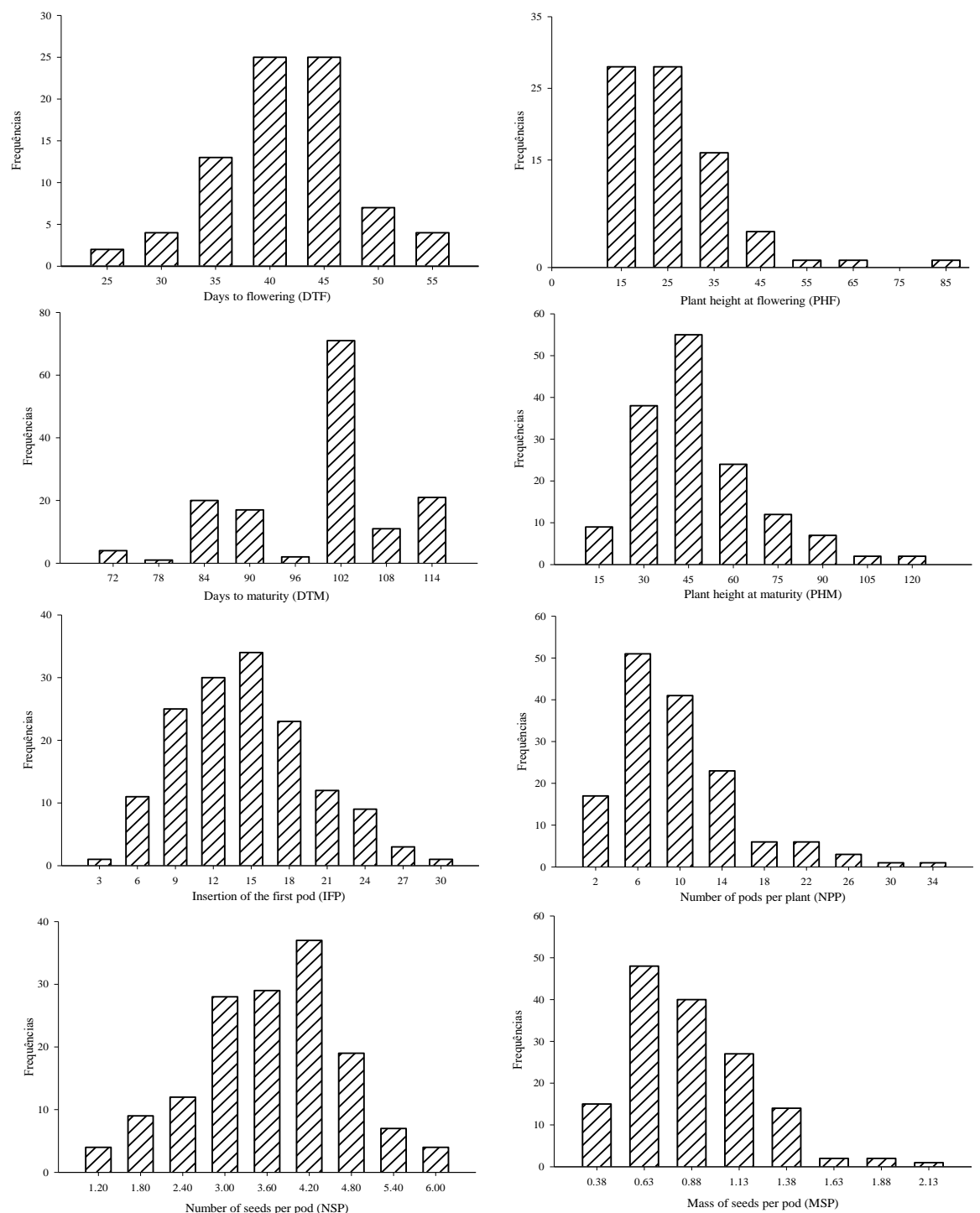

Fig 1. Distribution of frequencies of the phenotypic classes for, graph (A) days to flowering (DTF); graph (B) plant height at flowering (PHF); graph (C) days to maturity (DTM); graph (D) plant height at maturity (PHM); graph (E) insertion of the first pod (IFP); graph (F) number of pods per plant (NPP); graph (G) number of seeds per pod (NSP); graph (H) mass of seeds per pod (MSP), measured in the 2014/2015 crop.

cultivars BRS Esplendor and BRS Supremo, based on the standardized Euclidean distance (Table 2). This method has the purpose of subdividing the genotypes into groups, using the criterion of maintaining the lowest genetic dissimilarity within the group and the greatest dissimilarity between groups. In this way, genotypes have the lowest genetic distance form the first group (Cruz et al., 2012).

The genotypes were subdivided into eight groups. The group I consisting of 120 black bean accessions and the comercial cultivars BRS Esplendor and BRS Supremo, where the greatest genetic distances for group I were observed between the accessions 43 and 105. The group II consisted of 13 genotypes; group III, six genotypes; group IV three genotypes; group V two genotypes; and the groups VI, VII and VIII were consisted of only one genotype each. Research about 45 black bean genotypes showed great contribution of Tocher grouping to differentiate genotypes and direct future crossings between the groups more genetically distant (Elias et al., 2007).

Regarding the genetic distance between the groups (Table
3 ), higher means were observed between the groups $\mathrm{V}$ and VIII (2.27) and VI and VIII (2.21). Therefore, it is possible to increase the genetic variability of the breeding program, using the genotypes from more genetically distant groups. One alternative is to direct the crossings between the individuals of group I with group VIII (1.84), as in the first group there are accessions with agronomic ideotypes closer to the ideal phenotype of the commercial cultivars BRS Esplendor and BRS Supremo.

\section{Performance of traits for standardized Euclidean distance}

The standardized Euclidean distance is obtained through a collection of information measured by means of several characters evaluated in each individual (Cruz et al., 2012). The dendrogram shows the genetic dissimilarity between 147 accessions and the cultivars BRS Esplendor (148) and BRS Supremo (149). It was found that the accession 139 is characterized as the most dissimilar genotype, followed by 
Table 2. Results for the grouping of 147 common black bean accessions and controls BRS Esplendor and BRS Supremo, by the Tocher optimization method, based on the standardized Euclidean distance, considering of 16 characteristics of agronomic importance measured in the 2014/2015 crop.

\begin{tabular}{ll}
\hline Groups & Common black bean genotypes \\
\hline & $43,46,45,41,40,47,124,108,146,144,39,129,107,141,71,15,66,133$, \\
I & $42,69,1,12,123,38,145$, BRS Esplendor, $84,77,62,31,92,28,54,20,27,74$, \\
& $135,91,138,118,117,106,70,16,140,121,19,127,101,32,50,44,137,7$, \\
\hline & $52,58,14,37,18,119,8,34,96,128,72$, BRS Supremo, $57,132,82,93,13,89$, \\
& $116,109,5,3,30,63,49,88,130,25,48,80,120,75,125,61,76,68$, \\
& $65,21,126,23,131,142,78,73,22,53,67,24,6,59,83,2,10,35,60,99$, \\
& $90,143,134,29,9,11,95,79,122,33,100,105$ \\
II & $104,115,86,97,55,113,114,26,85,103,112,147,102$ \\
\hline III & $17,87,4,36,111,136$ \\
IV & $64,81,98$ \\
V & 94,110 \\
VI & 51 \\
VII & 56 \\
VIII & 139 \\
\hline
\end{tabular}
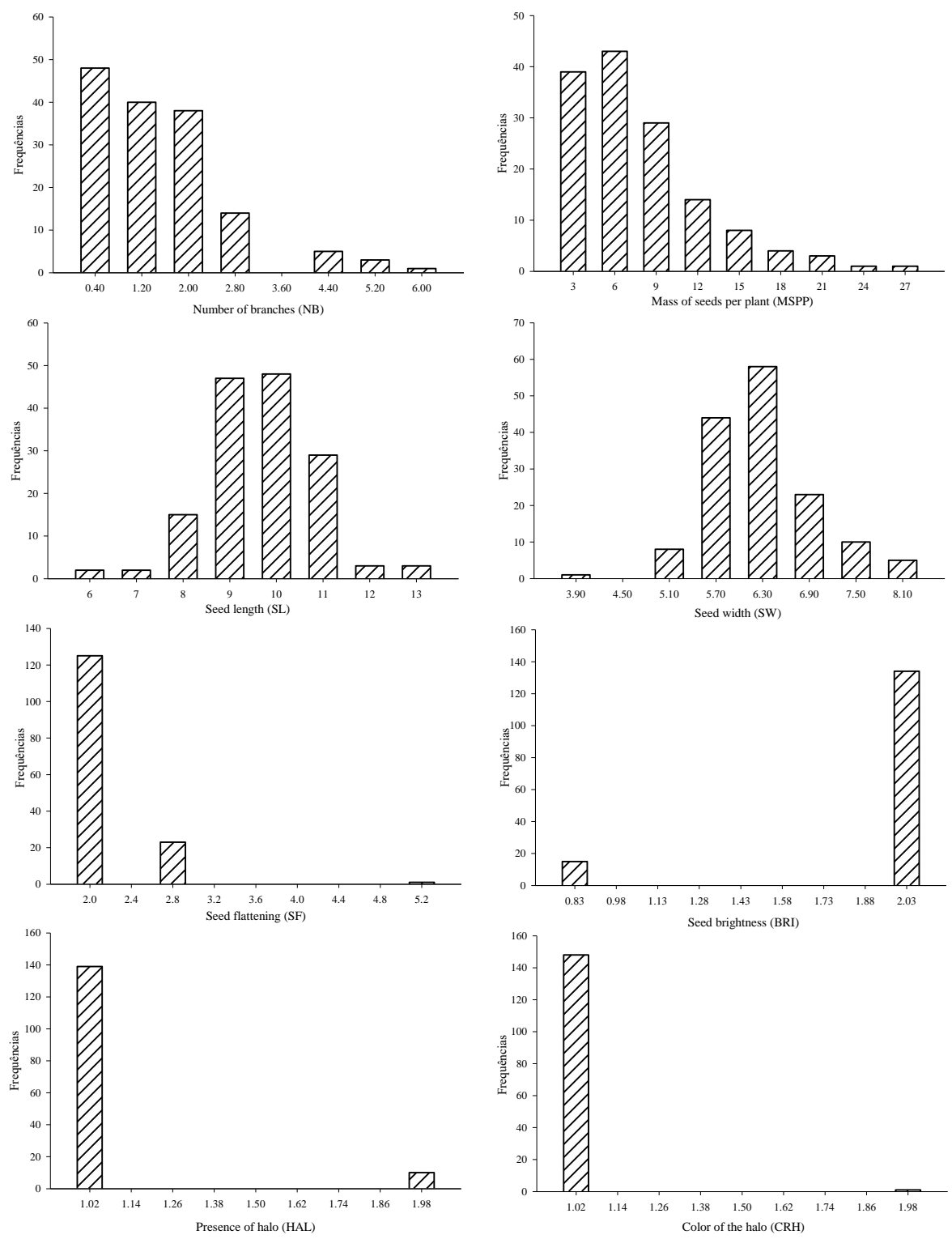

Fig 2. Distribution of frequencies of the phenotypic classes for, graph (A) number of branches (NB); graph (B) mass of seeds per plant (MSPP); graph (C) seed length (SL); graph (D) seed width (SW); graph (E) seed flattening (SF); graph (F) seed brightness (BRI); graph (G) presence of halo (HAL); graph (H) color of the halo (CRH), measured in the 2014/2015 crop. 
Table 3. Results of the average distance between groups estimated by the Tocher optimization method, involving 147 common black bean accessions and controls BRS Esplendor and BRS Supremo, considering 16 characteristics of agronomic importance measured in the 2014/2015 crop.

\begin{tabular}{|c|c|c|c|c|c|c|c|c|}
\hline Grupos & I & II & III & IV & V & VI & VII & VIII \\
\hline I & 0.80 & 1.35 & 1.23 & 1.38 & 1.27 & 1.33 & 1.29 & 1.84 \\
\hline II & & 0.93 & 1.49 & 1.56 & 1.65 & 1.74 & 1.66 & 1.56 \\
\hline III & & & 0.67 & 1.58 & 1.52 & 1.63 & 1.60 & 2.08 \\
\hline IV & & & & 0.92 & 1.45 & 1.61 & 1.73 & 2.07 \\
\hline V & & & & & 0.92 & 1.37 & 1.56 & 2.27 \\
\hline VI & & & & & & - & 1.51 & 2.21 \\
\hline VII & & & & & & & - & 2.05 \\
\hline VIII & & & & & & & & - \\
\hline
\end{tabular}

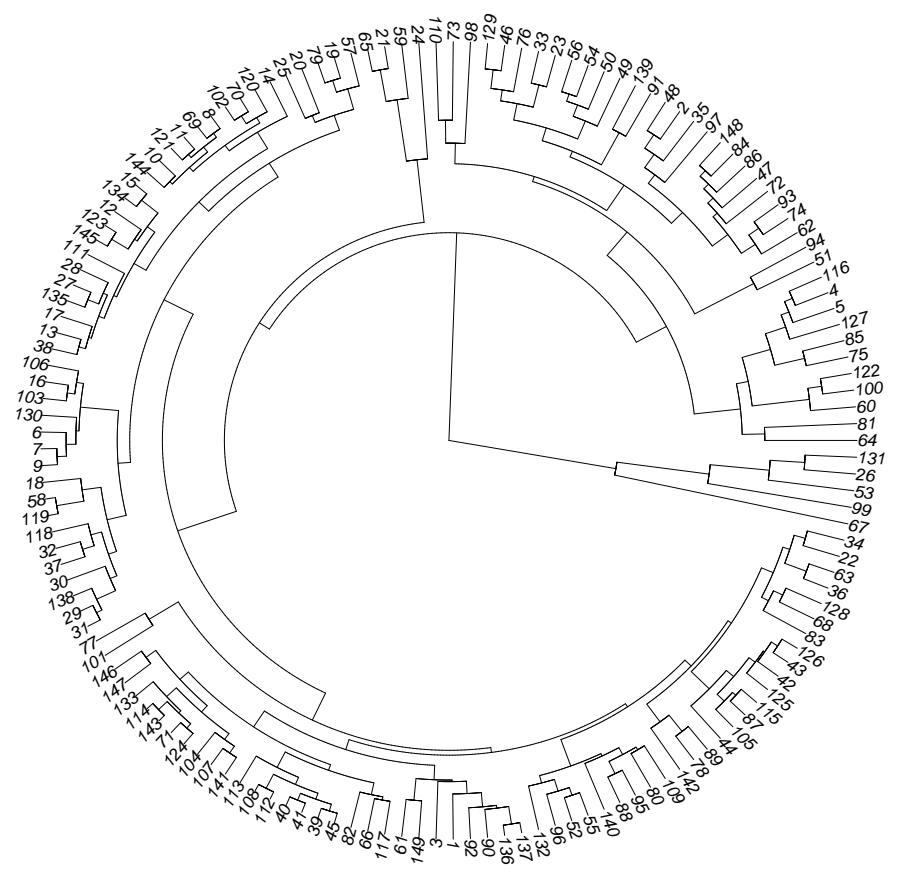

Fig3. Dendrogram with the genetic dissimilarity in 147 common black bean accessions and controls BRS Esplendor (148) and BRS Supremo (149), using the standardized Euclidean distance, obtained by the method of average connections (UPGMA), measured in the 2014/2015crop.

accessions 51 and 56 (Fig. 3). From these results, it is possible to identify that the grouping of unweighted pairs based on the arithmetic mean (UPGMA) shows similar results to those obtained by the Tocher optimization method, being possible to identify the formation of the eight groups, in which groups I, II, III IV, V, VI, VII and VIII are comprised of $122,13,6,3,2,1,1$, and 1 genotype, respectively. Comparison of clustering methods for bean genotypes concluded that the responses obtained by the standardized Euclidean distance are similar to those obtained by the Tocher optimization method (Cargnelutti Filho et al., 2008).

\section{Performance of traits for the relative contribution by Singh}

The relative contribution of the characters in the discrimination of genotypes was performed by Singh method (1981), using 16 characters measured in 147 accessions and two check cultivars, BRS Esplendor and BRS Supremo (Table 4). Thus, the variables with the highest relative contribution were days to flowering (DTF), with $21.93 \%$, and seed brightness (BRI) with $13.31 \%$. Studies in 57 black bean accessions concluded that the highest relative contributions to differentiate the genotypes are obtained by mass of hundred seeds, days to flowering and maturity, and seed size (Cabral et al., 2011).

\section{Performance of traits for the principal components}

The principal component analysis is characterized as a multivariate technique that allows, by means of a set of characters generating independent information, to explain and infer a response in the most informative way (Cruz et al., 2012). Given this, nine main components were needed to explain the genetic variation between the common black bean genotypes, where MC 1 explained (18.3\%), MC 2 (12.8\%), MC 3 (10.0\%), MC 4 (8.5\%), MC 5 (7.9\%), MC 6 (6.8\%), MC $7(6.1 \%)$, MC $8(5.9 \%)$ and MC $9(5.2 \%)$. These components together made it possible to explain $81.9 \%$ of all genetic variation involved in the 149 genotypes studied. 
Table 4. Results of the relative contribution of 147 common black bean accessions and controls BRS Esplendor and BRS Supremo, by the method of Singh (1981). Measured in the 2014/2015 crop.

\begin{tabular}{lcc}
\hline Variable & S.j & Relative Contribution (\%) \\
\hline Days to flowering (DTF) & 3303.72 & 21.93 \\
Plant height at flowering (PHF) & 958.34 & 6.36 \\
Days to maturity (DTM) & 343.51 & 2.28 \\
Plant height at maturity (PHM) & 701.24 & 4.65 \\
Insertion of the first pod (IFP) & 866.33 & 5.75 \\
Number of pods per plant (NPP) & 710.68 & 4.72 \\
Number of seeds per pod (NSP) & 947.64 & 6.29 \\
Mass of seeds per pod (MSP) & 688.61 & 4.57 \\
Number of branches (NB) & 1006.67 & 6.68 \\
Mass of seeds per plant (MSPP) & 730.60 & 4.85 \\
Seed length (SL) & 535.45 & 3.55 \\
Seed width (SW) & 430.59 & 2.86 \\
Seed flattening (SF) & 300.54 & 1.99 \\
Seed brightness (BRI) & 2005.00 & 13.31 \\
Presence of halo (HAL) & 1390.00 & 9.23 \\
Color of the halo (CRH) & 148.00 & 0.98 \\
\hline
\end{tabular}

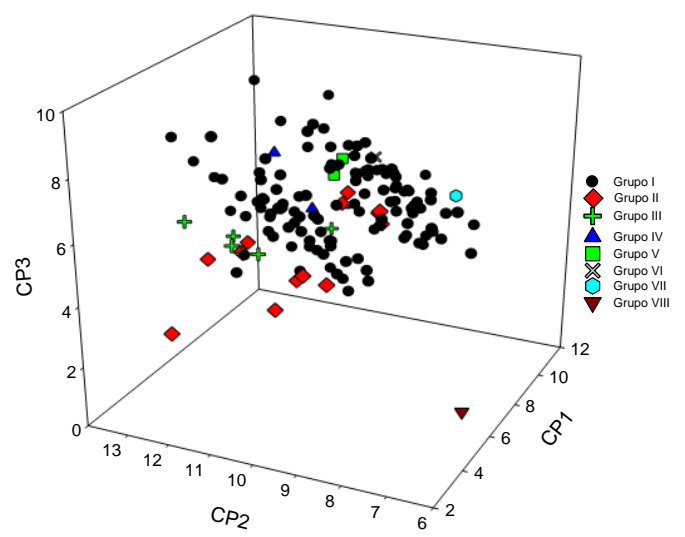

Fig 4. Results for the analysis of graphic dispersion of the scores in relation to the first three main components, MC1, MC2 and MC3, of the 147 common black bean accessions and controls BRS Esplendor and BRS Supremo, measured in the 2014/2015 crop.

Regarding the graphic dispersion of the accessions, only the three first main components (MC1, MC2, and MC3) were used to demonstrate the dispersion of genotypes (Fig 4). To better understand the distinction of genotypes, the Tocher optimization and classification was used, assigning distinct evidence to the eight formed groups. Based on the results obtained in each multivariate analysis, it was found that the response of the 147 accessions and the check cultivars (BRS Esplendor and BRS Supremo) are similar in Tocher optimization method, the standardized Euclidean distance and the principal component analysis. So, they were efficient to distinguish genotypes, being characterized as viable tools to assist in the selection of genotypes that will compose crossings blocks of a breeding program for the common black bean culture.

\section{Materials and Methods}

\section{Plant materials}

The experiment was conducted in the 2014/2015 crop season in FredericoWestphalen-RS, where the coordinates correspond to $27^{\circ} 39^{\prime}$ '56' S latitude and 53 $42^{\prime}$ ' $94^{\prime \prime}$ W longitude, with an altitude of 490 meters. The soil is classified as a Typic Alumino ferric Red Latosol and the climate is characterized, by Köppen, as subtropical ( $C f a$ ) (Moreno, 1961).

\section{Experimental design and experimental procedure}

The experimental design was an augmented blocks (RCBD) (Federer, 1956) with147 black common bean accesses, and two commercial cultivars witnesses (BRS Esplendor and BRS Supremo) with four repetitions, totally 149 treatments (each treatment as a one genotype).The accessions are from landraces of common black bean collected in different regions of southern Brazil, and available in the Laboratory of Genetic Breeding and Plant Production of the Federal University of Santa Maria Campus Frederico Westphalen, RS, Brazil (Table 1). The experimental units were composed of two lines spaced by 0.45 meters and two meters $(\mathrm{m})$ long, totally $1.80 \mathrm{~m}^{2}$. The population density employed for all accessions and cultivars of common black bean was of 10 seeds per linear meter, according to Cabral et al. (2011). The management was employed based on direct sowing, with fertilizer in the sowing line of $300 \mathrm{~kg} \mathrm{ha}^{-1}$ of the formulated N-P-K (10-20-20). A $90 \mathrm{~kg} \mathrm{ha}^{-1}$ of nitrogen was applied in the amide form. The control of weeds, insect pests and diseases were carried out, preventively.

\section{Traits measured}

The evaluated traits were: Days to flowering (DTF), measured by counting the number of days from seedling emergence until the issuance of the first flower bud. Plant 
height at flowering (PHF), measured from the ground level to the last fully expanded leaflet at the beginning of the reproductive period. Days to maturity (DTM) was measured by counting the number of days from seedling emergence to harvest. Plant height at maturity (PHM) was measured from the ground level to the apex of the plant measured at harvest. Insertion of the first pod (IFP) was measured by the distance between the ground level and the insertion of the first pod viable on the main stem. Number of pods per plant (NPP) was determined by counting the total number of viable pods per plant. Number of seeds per pod (NSP) was obtained by the ratio between the total number of viable pods and the number of seeds per plant, results in units. Mass of seeds per pod (MSP) was obtained by the ratio between the total mass of seeds of the plant and the number of viable pods, results in grams (g). Number of branches (NB) was counted of the number of branches larger than ten centimeters. Mass of seeds per plant (MSPP) was the pods harvested on each plant were threshed, subsequently it was determined the humidity degree and the mass of seeds was adjusted to $13 \%$ moisture. Seed length (SL) was measured by the length of all plant seeds, character determined by using a digital caliper. Seed width (SW) was measured by the width of all plant seeds, character determined through a digital caliper. Seed flattening (SF) was determined by the methodology proposed by Silva (2005), wherein seeds are classified as flattened, semiflattened and filled. Seed Brightness (BRI) was conducted by visual grading through the methodology of Silva (2005), wherein seeds are classified as opaque, intermediate and bright. Presence of halo (HAL) was visual assessed determining the presence or absence of halo in the seed. Color of the halo (CRH) was visual assessment determining the color of the halo in relation to the seed.

\section{Statistical analysis}

The data obtained were submitted to descriptive analysis. Thereafter, the distributions of phenotypic frequencies of the traits were performed. Analysis of genetic dissimilarity between genotypes by standardized Euclidean distance was subsequently carried out, applying the UPGMA (unweighted pair grouping method with arithmetic mean). The relative importance of the characters was obtained by the methodology proposed by Singh (1981). The Tocher optimization method was held based on the matrix of the standardized Euclidean distance, and then the principal component analysis was held. All analyses were performed using the Genes software (Cruz, 2013).

\section{Conclusions}

The distributions of phenotypic frequencies indicate genetic variability among the 149 genotypes of common black bean. The Tocher optimization method presents the formation of eight groups of genotypes. The dendrogram formed by the standardized Euclidean distance was efficient in the stratification of accessions for their genetic distance. The relative contribution by Singh method shows that days to flowering and seed brightness are the characters that best discriminate genotypes. The multivariate techniques of Tocher optimization and standardized Euclidean distance show similar responses, proving to be viable tools for the choice of parents in a genetic breeding program.

\section{Acknowledgements}

The frist author acknowledges a fellowship by Universidade
Federal de Santa Maria Campus Frederico Westphalen (UFSM) Brazil, and Laboratório de Melhoramento Genético e Produção de Plantas (UFSM).

\section{References}

Cabral PDS, Soares TCB, Lima ABP, Alves DS, Nunes JA (2011) Genetic diversity of common bean accesses for agronomic traits. Rev Ciên Agro. 42:898-905.

Carbonell SAM, Carvalho CRL, Pereira, VR (2003) Technological quality of grain grown bean genotypes in different environments. Bragantia. 62:369-379.

Cargnelutti Filho A, Ribeiro NDR, Reis RC, Souza JR, Joast E (2008) Comparison of clustering methods for the study of genetic diversity in common bean cultivars. Ciên Rural. 38:2138-2145.

Coimbra JLM, Guidolin AF, Carvalho FIF, Azeved R (2000) Correlations canonical II - Analysis of yield beans and their components. Ciên Rural. 30:31-35.

Cruz CD (2013) Genes a software package for analysis in experimental statistics and quantitative genetics. Act Sci Agron. 35:271-276.

Cruz CD, Regazzi AJ, Carneiro PCS (2012) Biometric templates applied to breeding. 2ed. UFV Viçosa. 514p.

Elias HT, Vidigal MCG, Gonela A, Vogt GA (2007) Genetic variability in traditional black bean germplasm in Santa Catarina. Pesq Agrop Bras. 42:1443-1449.

Federer WT (1956) Augmented (hoonuiaku) designs. Haw Plan Rec. 55:191-208.

Florez A, Pujolà M, Valero J, Centelles E, Almirall A, Casañas F (2009) Genetic and environmental effects on chemical composition related to sensory traits in common beans (Phaseolus vulgaris L.). Food Chem. 113:950-956.

Horn F L, Schuch LOB, Silveira EA, Antunes IF, Vieira JC, Marchioro G, Medeiros DF, Schwengber JE (2000) Spacings assessment and populations of bean plants aiming the direct mechanical harvesting. Pesq Agrop Bras. 35:41-46.

Kurek AJ, Carvalho FIF, Assann I, Marchioro VS, Cruz PJ (2001) Path analysis as a criterion for indirect selection for grain yield in beans. Rev Bras Agroc. 7:29-32.

Marin A, Cervigni G D, Moreira M A, Barros E G (2005) Selection assisted by molecular markers for the development of plants resistant to disease, with emphasis on beans and soybeans. Fito Bras. 30:333-342.

Morais LK, Carbonell SAM, Pinheiro JB, Fonseca NS, Brasil EM (2001) Bean cultivars evaluation, Phaseolus vulgaris L. under different spacings. Acta Scien. 23:1199-1203.

Moreno JA (1961) Climate Rio Grande do Sul, Porto Alegre. $42 \mathrm{p}$.

Ramos Junior EU, Lemos LB, Silva TRB (2005) Yield components, grain yield and technological characteristics of common bean cultivars. Bragantia. 64:75-82

Rocha F, Stinghen JC, Gemeli MS, Coimbra JLM, Guidolin AF (2014) Diallel analysis as a tool in selecting parents in beans. Rev Ciên Agron. 45:74-81.

Ramalho MAP, Abreu AFB, Santos JB, Nunes JAR (2012) Applications of quantitative genetics in breeding of autogamous plants. Ed. Lavras: UFLA. 522p.

Silva FB, Ramalho MAP, Abreu AFB (2007) Phenotypic recurrent selection for early flowering bean 'Carioca'. Pesq Agrop Bras. 42:1437-1442.

Silva HT (2005) Minimum descriptors indicated to characterize cultivars/common bean varieties (Phaseolus vulgaris L.). Inf Téc Embrapa. 30p.

Singh D (1981) The relative importance of characters affecting genetic divergence. Ind J Gen Plant Breed. 41:237-245. 